\title{
Inhibition of STAT3 activity delays obesity-induced thyroid carcinogenesis in a mouse model
}

\author{
Jeong Won Park, Cho Rong Han, Li Zhao, Mark C Willingham and Sheue-yann Cheng
}

Laboratory of Molecular Biology, Center for Cancer Research, National Cancer Institute, National Institutes of Health, 37 Convent Dr, Room 5128, Bethesda, Maryland 20892-6264, USA
Correspondence should be addressed to S Cheng

Email chengs@mail.nih.gov

\begin{abstract}
Compelling epidemiologic studies indicate that obesity is a risk factor for many human cancers, including thyroid cancer. In recent decades, the incidence of thyroid cancer has dramatically increased along with a marked rise in obesity prevalence. We previously demonstrated that a high fat diet (HFD) effectively induced the obese phenotype in a mouse model of thyroid cancer (Thrb ${ }^{\text {PVIPV }}$ Pten $^{+/-}$mice). Moreover, HFD activates the STAT3 signal pathway to promote more aggressive tumor phenotypes. The aim of the present study was to evaluate the effect of S3I-201, a specific inhibitor of STAT3 activity, on HFD-induced aggressive cancer progression in the mouse model of thyroid cancer. WT and Thrb ${ }^{P V I P V}{ }^{P t e n}{ }^{+I-}$ mice were treated with HFD together with S3I-201 or vehicle-only as controls. We assessed the effects of S3I-201 on HFD-induced thyroid cancer progression, the leptin-JAK2-STAT3 signaling pathway, and key regulators of epithelial-mesenchymal transition (EMT). S3I-201 effectively inhibited HFD-induced aberrant activation of STAT3 and its downstream targets to markedly inhibit thyroid tumor growth and to prolong survival. Decreased protein levels of cyclins D1 and B1, cyclin dependent kinase 4 (CDK4), CDK6, and phosphorylated retinoblastoma protein led to the inhibition of tumor cell proliferation in S3I-201-treated Thrb $^{\text {PVIPV }}$ Pten $^{+/-}$mice. Reduced occurrence of vascular invasion and blocking of anaplasia and lung metastasis in thyroid tumors of S3I-201-treated Thrb ${ }^{P V I P V}$ Pten $^{+/-}$mice were mediated via decreased expression of vimentin and matrix metalloproteinases, two key effectors of EMT. The present findings suggest that inhibition of the STAT3 activity would be a novel treatment strategy for obesity-induced thyroid cancer.
\end{abstract}
Key Words
- thyroid cancer
- JAK2-STAT3 signaling
- obesity
- STAT3 inhibitor
- preclinical mouse model
- Pten-deficiency
- thyroid hormone receptor $\beta$ mutant

Endocrine-Related Cancer (2016) 23, 53-63

\section{Introduction}

Thyroid cancer is the most common malignancy in the endocrine organs. Over the past several decades, the incidence of thyroid cancer has been increasing faster than that of any other cancer. At the same time, the rates of obesity and metabolic syndrome have also risen, and accumulating evidence indicates a close association of obesity with increased cancer risk. Numerous epidemiological studies suggest that obesity is independently associated with an increased incidence of various solid tumors, including thyroid cancer (Renehan et al. 2008).

Recent studies showed that the risk of thyroid cancer was significantly increased by excess body weight, either overweight or obesity (Meinhold et al. 2010, AschebrookKilfoy et al. 2011, Kitahara et al. 2011, Simard et al. 2012, Zhao et al. 2012, Pappa \& Alevizaki 2014). In a retrospective study of 2057 patients with papillary thyroid

Published by Bioscientifica Ltd. 
cancer (PTC), Kim et al. (2013a) found that a higher BMI was correlated with a more aggressive PTC phenotype, such as increased tumor size, extrathyroidal invasion, and advanced disease stage, independent of age, sex, and other confounding factors. While compelling epidemiological data support the positive correlation of obesity with increased risk thyroid cancer, the molecular mechanisms by which obesity increase the risk of thyroid cancer progression are poorly understood.

Because it would be difficult to study how obesity could affect thyroid carcinogenesis in patients at the molecular level, we have recently used mouse models of thyroid cancer (Thrb ${ }^{P V / P V}$ Pten $^{+/-}$mice) to elucidate the underlying mechanisms (Kim et al. 2013b). $\mathrm{Thrb}^{\text {PV/PV }}$ Pten $^{+/-}$mice express a potent dominantly negative thyroid hormone receptor $\beta$ (TR $\beta \mathrm{PV}$ ) and haploinsufficiency in the Pten gene (phosphatase and tensin homolog deleted from chromosome 10). As Thrb ${ }^{P V / P V}{ }$ Pten $^{+/-}$mice age, they spontaneously develop aggressive follicular thyroid cancer similar to human cancer (Guigon et al. 2009). Treatment of these mice with a high fat diet (HFD) led to obesity with increased weight of body and fat tissues, enlarged adipocytes, and markedly elevated serum leptin levels (Kim et al. 2013b). Biochemical and histopathological analyses showed that an HFD induces more aggressive pathological changes with increased tumor cell proliferation, shortened survival, and frequent occurrence of anaplasia. Molecular analyses revealed that over-activated leptin-Janus kinase 2 (JAK2)-STAT3 signaling increases expression of STAT3 downstream target genes to drive HFD-induced thyroid cancer progression in Thrb ${ }^{\text {PV/PV }} \mathrm{Pten}^{+/-}$(Kim et al. 2013b). These findings suggested that STAT3 could be tested as a potential molecular target for the obesity-induced thyroid cancer.

Accordingly, we hypothesized that inhibition of STAT3 activity could delay obesity-induced thyroid carcinogenesis. To test this hypothesis, we treated $\mathrm{Thrb}^{\text {PV/PV }} \mathrm{Pten}^{+/-}$mice with an HFD in the presence or absence an STAT3-specific inhibitor, S3I-201. This inhibitor, which was identified through structure-based virtual screening of the National Cancer Institute libraries, has been shown to be effective in the inhibition of STAT3 activity by in vitro and in vivo studies (Siddiquee et al. 2007, Sen et al. 2012). We monitored the effect of S3I-201 on survival of these mice, tumor growth, tumor cell invasion, and metastasis. Indeed, we found that S3I-201 was effective in the inhibition of STAT3 activity, leading to delayed thyroid cancer progression and blocking of metastatic spread induced by an HFD.

\section{Materials and methods}

\section{Animals and treatment}

The National Cancer Institute Animal Care and Use Committee approved the protocols for animal care and handling in the present study. Mice harboring the ThrbPV gene $\left(T h r b^{P V / P V}\right.$ mice) were prepared via homologous recombination, and genotyping was carried out using the PCR method, as previously described (Guigon et al. 2009). Pten $^{+/-}$mice were kindly provided by Dr Ramon Parsons (Columbia University, NY, USA). Thrb ${ }^{P V / P V}$ Pten $^{+/-}$ mice were obtained by first crossing Pten $^{+/-}$mice with $T h r b^{P V / P V}$ mice and then crossing Thrb ${ }^{P V /+} \mathrm{Pten}^{+/-}$with $\mathrm{Thrb}^{\mathrm{PV} /+} \mathrm{Pten}^{+/-}$mice. The HFD (60\% calories from fat) were purchased from Research Diets (New Brunswick, NJ, USA). The mice were administered HFD diet from the age of 8 weeks until the end of the study. S3I-201 (STAT3 inhibitor, cat\# S1155, Selleckchem, Huston, TX, USA) was dissolved in a solution of DMSO (0.05\% DMSO) and injection by i.p. three times a week at a dose of $5 \mathrm{mg} / \mathrm{kg}$ per mouse or vehicle $(0.05 \%$ DMSO) starting at the age of 8 weeks. They were monitored until they became moribund with hunched posture and labored breathing. After the mice were euthanized, the thyroids and lungs were dissected for weighing, histologic analysis, and biochemical studies.

\section{Hormone assay}

Serum TSH levels were measured as previously described (Furumoto et al. 2005). Serum levels of total $\mathrm{T}_{3}$ and $\mathrm{T}_{4}$ were determined by commercialized radioimmunoassay (RIA) kits (cat. 06B256447 and 06B254029) from MP Biomedical, LLC. Serum levels of leptin of the mice were measured by using a leptin RIA kit (cat. XL-85K, Billeria, MA, USA) from Millipore Corporation.

\section{Histopathologic analysis}

Thyroid glands, lungs, and inguinal fat were dissected and fixed in 10\% neutral-buffered formalin (Sigma-Aldrich) and subsequently embedded in paraffin. Five-micrometerthick sections were prepared and stained with hematoxylin and eosin. For each animal, single random sections of thyroid and lung were examined. For thyroids, morphologic evidence of hyperplasia, capsular invasion, and vascular invasion was routinely examined in that single section. The presence of a single microscopic focus of metastatic follicular carcinoma in the lung was counted

Published by Bioscientifica Ltd 
as a metastatic lesion in that animal. Adipocyte size (expressed as an average area per cell $\left(\mu \mathrm{m}^{2} / 1 \times 10^{-3}\right.$ cells)) was calculated based on the number of adipocytes in the same field size.

Immunohistochemistry (IHC) was conducted as previously described with some modifications (Zhu et al. 2014). For the antigen retrieval step, slides were heated in $0.05 \%$ citraconic anhydride solution (Sigma-Aldrich; $\mathrm{pH} 7.4$ ) at $98^{\circ} \mathrm{C}$ for $60 \mathrm{~min}$ followed by treatment with antiKi-67 antibody (dilution 1:300, cat. RB-9043-P0, Thermo Scientific, Cabmbrige, MA, USA) and rabbit anti-p-STAT3 antibody (1:100 dilution, Cell Signaling, Denver, MA, USA) at $4{ }^{\circ} \mathrm{C}$ overnight. The antigen signals were detected by treatment with the peroxidase substrate diaminobezidine followed by counterstaining with Gill's hematoxylin (Electron microscopy sciences, Hatfiled, PA, USA). Relative positive cell ratio was quantified by using NIH IMAGE software (Image J 1.47).

\section{Western blot analysis}

Preparation of whole-cell lysates from thyroid glands has been described previously (Zhu et al. 2014). The protein sample $(30 \mu \mathrm{g})$ was loaded and separated by SDS-PAGE. After electrophoresis, the protein was electrotransferred to a poly vinylidenedifluoride membrane (Immobilon-P; Millipore Corp., Billeria, MA, USA). The antibodies phosphorylated $\mathrm{Rb}$ (p-Rb, S780, 1:500 dilution), total-Rb (1:1000 dilution), Cdk4 (1:1000 dilution), Cdk6 (1:1000 dilution), p-Jak2 (Y1007/1008, 1:500 dilution), total-Jak2 (1:1000 dilution), p-STAT3 (1:500 dilution), total-STAT3 (1:1000 dilution), glyceraldehyde-3-phosphate dehydrogenase (GAPDH; 1:1000 dilution), and vimentin (1:1000 dilution) were purchased from Cell Signaling Technology. Antibody for MMP-2 (1:200 dilution) and cyclin B1 (1:200 dilution) were purchased from Santa Cruz Biotechnology (Dallas, TX, USA). Antibody for cyclin D1 (1:200 dilution) and leptin receptor $(1: 2,000$ dilution) were purchased from Neomarkers and Abcam respectively. The blots were stripped with Re-Blot Plus (Millpore, Billeria, MA, USA) and reprobed with rabbit polyclonal antibodies to GAPDH. Band intensities were quantified by using NIH IMAGE software (Image J 1.47).

\section{Quantitative real-time RT-PCR}

Total RNA was extracted from thyroid tumor of mice using TRIzol (Invitrogen) according to the manufacturer's protocol. For quantitative real-time RT-PCR (qRT-PCR), one-step RT-PCR reactions were performed with $200 \mathrm{ng}$ of total RNA using a QuantiTect SYBR green RT-PCR kit (Qiagen) in 7900HT Fast Real-time PCR system (Applied Biosystems) according to the manufacturer's instructions.

\section{Statistical analysis}

All data are expressed as mean \pm s.E.M., and Student's $t$-test was used to compare continuous variables accordingly. The Kaplan-Meier method with log-rank test was used to compare survival in each treatment group. Statistical significance was set at $P<0.05$. GraphPad Prism 6.0 (GraphPad Software, La Jolla, CA, USA) was used to draw graphs.

\section{Results}

S3I-201 reduces thyroid tumor growth and increases survival in HFD-induced obese $\mathrm{Thrb}^{\mathrm{PV} / \mathrm{PV}} \mathrm{Pten}^{+/-}$mice

Previously, we reported that the diet-induced obesity in $\mathrm{Thrb}^{\text {PV/PV }}$ Pten $^{+/-}$mice promoted thyroid carcinogenesis by reducing survival rate, increasing tumor growth and advancing tumor stages in $T h r b^{P V / P V}{ }^{P_{t e n}{ }^{+/-}}$mice. We elucidated that one of the pathways in the obesityinduced tumor progression was via activation of the leptin-JAK2-STAT3 signaling pathway (Kim et al. 2013b).

To test whether STAT3 could serve as a potential molecular target, we evaluated the effect of an STAT3specific inhibitor, S3I-201, on thyroid carcinogenesis of HFD-treated $\mathrm{Thrb}^{P V / P V}$ Pten $^{+/-}$mice. Figure 1A shows that compared with vehicle-treated mice (open squares), the $50 \%$ survival age of S3I-201-treated mice was significantly increased by 1 month (closed squares). Moreover, analysis of thyroid tumor weight of HFD-treated Thrb ${ }^{P V / P V}$ Pten $^{+/-}$ mice indicated a significant reduction by S3I-201 treatment (data set 4 vs data set 3, $n=11-13, P<0.05$ ) (Fig. 1B). A small decrease was also observed in the normal thyroid of WT mice (data set 2 vs data set $1, n=10-11, P<0.05$ ). That the proliferation of tumor cells was inhibited by S3I201 was further shown by immunohistochemical analysis using Ki-67 as a proliferation marker (Fig. 1C-I). Compared with the negative controls (panels a and c) a reduction in Ki-67 positively-stained cells was detected in the thyroid sections of HFD-WT mice treated with S3I-201 (panel d) compared with HFD-WT mice treated with vehicle only (panel b). However, many more hyperplastic tumor cells intensely stained with Ki-67 were detected in the thyroid tumor section of HFD-Thrb ${ }^{P V / P V}$ Pten $^{+/-}$mice treated with vehicle (compare panel $f$ with panel b). Markedly fewer tumor cells stained with Ki-67 were detected in

Published by Bioscientifica Ltd. 
A

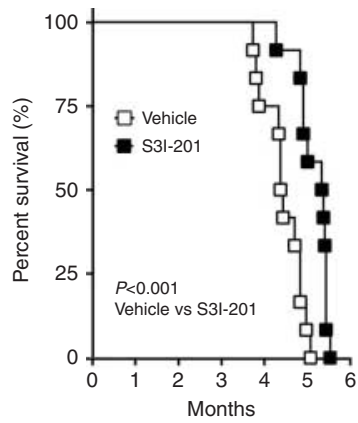

B

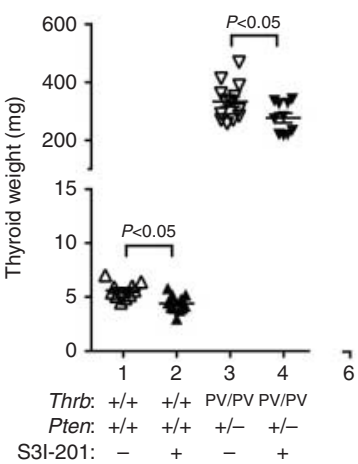

C-I

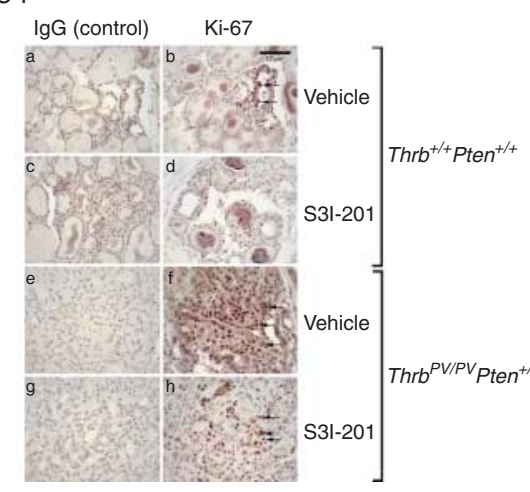

C-II

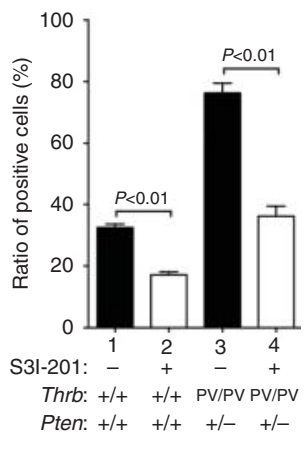

\section{Figure 1}

The effects of S3I-201 on thyroid tumor growth and survival of HFD-WT and HFD-Thrb ${ }^{\text {PVIPV }}$ Pten ${ }^{+/-}$mice. (A) Survival curves for HFD-Thrb ${ }^{\text {PVIPV }}$ Pten $^{+/-}$ mice treated with S3I-201 (5 mg/kg body weight, $n=12)$ or vehicle $(0.05 \%$ DMSO, $n=12)$ ) i.p. injection three times a week from 8 weeks of age until they had to be euthanized because of sickness. Data are presented by Kaplan-Meir methods and analyzed by log-rank test. The $P$ values are indicated. (B) Thyroid weights of vehicle-treated or S3I-201-treated WT ( $n=10-11)$ or Thrb $^{\text {PVIPV }}$ Pten $^{+/-}$mice $(n=10-13)$. (C-I). Comparison between the two groups of representative microphotographs of immunohistochemistry $(\mathrm{IHC})$ one using anti-Ki67 antibody on thyroid sections

HFD-Thrb ${ }^{P V / P V}$ Pten $^{+/-}$mice (panel h). Panels e and g are the corresponding negative controls.

The comparison can be seen more clearly by the quantitative analysis shown in Fig. 1C-II. More tumor cells (1.7-fold) were intensely stained with Ki-67 in HFD-treated $\mathrm{Thrb}^{\mathrm{PV} / \mathrm{P} V} \mathrm{Pten}^{+/-}$mice (bar 3) than in follicular cells of WT mice. It is important to note that a marked 30\% reduction of Ki-67 positively-stained cells was found in the thyroid sections of inhibitor-treated Thrb ${ }^{P V / P V}$ Pten $^{+/-}$mice (bar 4) as compared with vehicle-treated mice (bar 3). In the HFDWT mice, the effect of S3I-201 was a 50\% reduction in cell proliferation (bars 2 vs 1). Taken together, these data indicated that treatment of HFD-Thrb ${ }^{P V / P V}$ Pten $^{+/-}$mice with S3I-201 delayed thyroid growth and increased survival.

\section{An STAT3 inhibitor, S3I-201, reduces thyroid tumor cell proliferation in HFD-induced obese Thrb $^{\text {PV/PV }}$ Pten $^{+/-}$mice}

It is known that TSH is a major stimulator of thyrocyte proliferation. To evaluate whether the decreased thyroid tumor growth of HFD-Thrb ${ }^{P V / P V}$ Pten $^{+/-}$mice treated with S3I-201 inhibitor could possibly result from lower TSH levels, we compared serum $\mathrm{TSH}$, total $\mathrm{T}_{4}$, and total $\mathrm{T}_{3}$ levels. We found no significant differences in the levels of serum $\mathrm{TSH}$, total $\mathrm{T}_{4}$, and total $\mathrm{T}_{3}$ between S3I-201 treatment and vehicle treatment in both WT and from WT mice with/without S3I-201 (panels a, b, c, and d) and one without the treatment with primary antibodies are shown in the corresponding panels ( $a$ and $c$ ). The thyroid sections from $T_{h r b^{P V I P V}}$ ten $^{+/-}$mice (e, f, g, and $h$ ) and the negative without the treatment with primary antibodies are shown in the corresponding panels (e and g). (C-II) The Ki67-positive cells were counted and the data expressed as percentage of Ki67-positive cells vs total cells. The data are expressed as mean \pm S.E.M. $(n=2$ slides). The $P$ values are shown. A full colour version of this figure is available at http://dx.doi. org/10.1530/ERC-15-0417.

$T r b^{P V / P V}$ Pten $^{+/-}$mice. These data indicated that the reduced thyroid tumor growth in S3I-201-treated Thrb $^{P V / P V}$ Pten $^{+/-}$mice was not due to the effects of TSH and thyroid hormone $\left(\mathrm{T}_{3}\right.$ and $\left.\mathrm{T}_{4}\right)$.

To understand how S3I-201 reduced cell proliferation in HFD-Thrb ${ }^{P V / P V}$ Pten $^{+/-}$mice, we evaluated the abundance of key regulators in the cell cycle progression with or without the inhibitor. The protein levels of cyclin D1 and cyclin B1 were lower in the S3I-201-treated HFDThrb $^{P V / P V}$ Pten $^{+/-}$mice than the vehicle-treated group (Fig. 2A-a and -b). In addition, the protein levels of cyclin dependent kinase 4 (CDK4) and CDK6 (CDK6) were lower in the S3I-201-treated HFD-Thrb ${ }^{P V / P V}$ Pten $^{+/-}$mice than the vehicle-treated group (Fig. 2A-c and d). The increased abundance of these cyclins and their kinases led to an increased phosphorylated retinoblastoma ( $\mathrm{Rb}$ ) (Fig. 2A-e) without apparent changes in total $\mathrm{Rb}$ protein levels (Fig. 2A-f) to drive cell cycle progression from the G1 phase to the $S$ phase (Nevins 1992). Panel g shows the loading control using GAPDH. The intensities of the bands in Fig. 2A were quantified to demonstrate the significant decreases in cyclin D1 (panel a), B1 (panel b), CDK4 (panel c), and CDK6 (panel d) and the ratios of phosphorylated $\mathrm{Rb}$ vs total $\mathrm{Rb}$ (panel e) in thyroid tumors of inhibitortreated HFD-Thrb ${ }^{P V / P V}$ Pten ${ }^{+/-}$mice. Some minor decreases of CDK4 protein levels (panel c) and ratios of phosphorylated $\mathrm{Rb}$ vs total $\mathrm{Rb}$ were also detected in

Published by Bioscientifica Ltd 
A

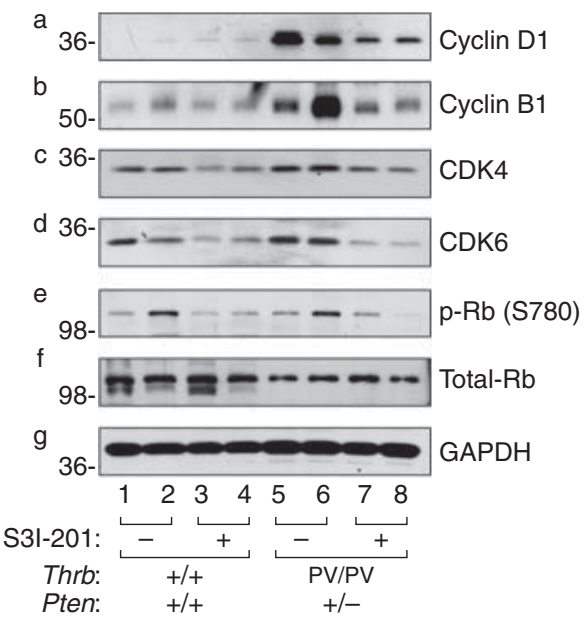

B

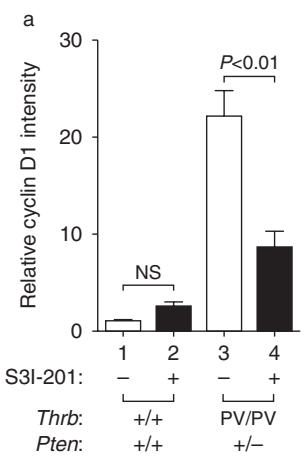

d

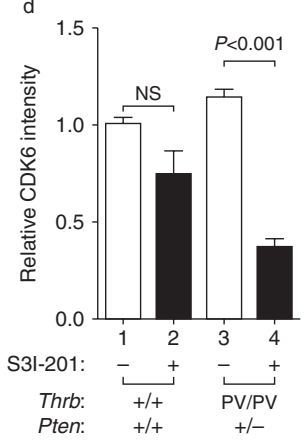

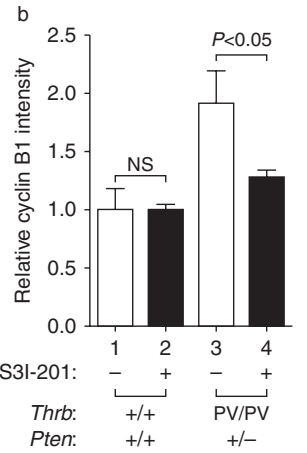
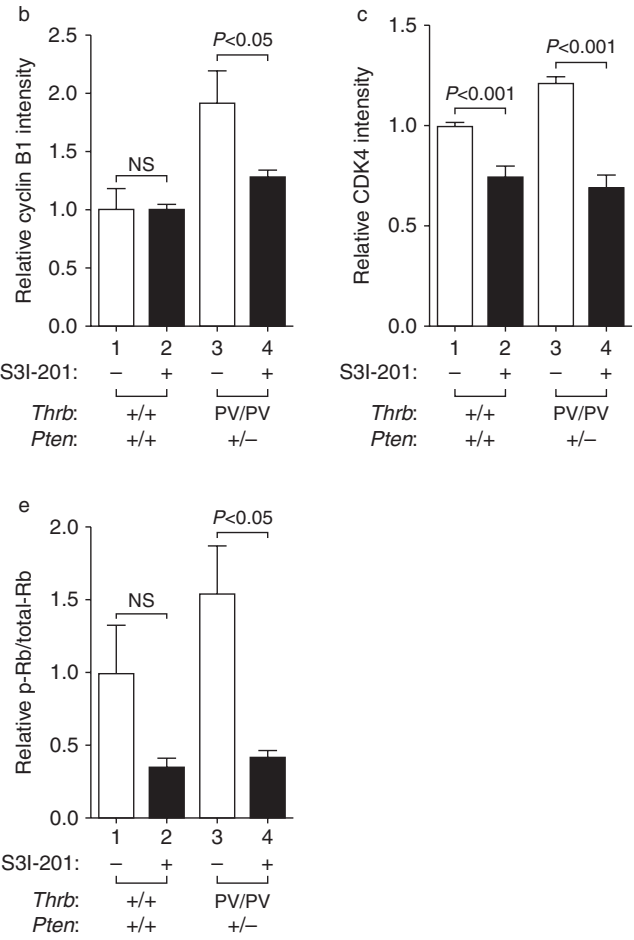

\section{Figure 2}

The effects of S3I-201 on the protein levels of key regulators of the cell cycle in thyroid tumors from HFD-WT and HFD-Thrb ${ }^{P V / P V}$ Pten $^{+1-}$ mice.

(A) western blot analysis of cyclin D1, cyclin B1, cdk4, cdk6, phosphorylated retinoblastoma ( $\mathrm{p}-\mathrm{Rb}$; $\mathrm{S780}$ ), and total $\mathrm{Rb}$ in mice treated with vehicle (lanes 1, 2, 5, and 6) or with S3I-201 (lanes 3, 4, 7, and 8). Two mice were used in each group. GAPDH was used as a loading control (panel g). (B) The band intensities of the protein detected in A were quantified and compared. The filled bars were from mice treated with the inhibitor, S3I-201, and the open bars are from mice treated with vehicle. The data, shown as mean \pm S.E.M., were analyzed by Student's $t$-test. thyroid of inhibitor-treated WT mice. These data indicate that treatment of HFD-Thrb ${ }^{P V / P V}{ }^{P t e n}{ }^{+/-}$mice with S3I201 led to the reduction of the key cell cycle regulators to inhibit the proliferation of tumor cells.

\section{An STAT3 inhibitor, S3I-201, inhibits STAT3 activity in HFD-induced obese thyroid cancer Thrb $^{\text {PV/PV }}$ Pten $^{+/-}$mice to delay tumor progression and prevent metastasis}

Previously we showed that $T h r b^{P V / P V}$ Pten $^{+/-}$mice treated with HFD resulted in a fivefold elevated serum leptin level (Kim et al. 2013b). In this study, as a control, we assessed whether serum leptin levels of HFD-Thrb ${ }^{P V / P V}$ Pten $^{+/-}$mice were affected by S3I-201 treatment. Figure 3A shows that S3I-201 treatment led to significant decreases in serum leptin levels of HFD-Thrb ${ }^{P V / P V}$ Pten $^{+/-}$mice (data set 3 vs set 4). However, no apparent effect of the inhibitor on the serum levels of HFD-WT mice was observed (data set 1 vs set 2). The elevated serum leptin levels promoted us to examine the histology of the white fat tissue. Consistent with decreased leptin levels in HFD-Thrb ${ }^{P V / P V}$ Pten $^{+/-}$mice treated with S3I-201, we found that enlarged inguinal fat cell size of HFD-Thrb ${ }^{P V / P V}$ Pten $^{+/-}$mice (9; panel c, Fig. 3B-I) was decreased in mice treated with S3I-201 (panel d, Fig. 3B-I). Quantitative analysis shows that the size of the adipocytes of HFD-Thrb ${ }^{P V / P V}$ Pten $^{+/-}$mice treated with S3I-201 was 70\% smaller than that of the fat cells of the vehicle-treated HFD-Thrb ${ }^{P V / P V}{ }^{\text {Pten }}{ }^{+/-}$mice (bars 4 vs 3, Fig. 3B-II). However, no effect of the inhibitor was detected in the fat cell size of HFD-WT mice (panel a vs b; Fig. 3B-I and bars 1 vs 2, Fig. 3B-II).

Using western blot analysis, we next examined whether the protein abundance of the leptin receptor was altered in the thyroid of mice treated with S3I-201. Figure 4A-a shows that the protein abundance of the leptin receptor was higher in the thyroid of HFD-Thrb ${ }^{\text {PV/PV }}$ Pten $^{+/-}$ mice (lanes 5-8, Fig. 4A-a) than that of HFD-WT mice (lanes 1-4, Fig. 4A-a). However, no effect of S3I-201 on the leptin receptor was detected either in HFDThrb $^{\text {PV/PV }}$ Pten $^{+/-}$mice (lanes 5 and 6 vs lanes 7 and 8 ) or

Published by Bioscientifica Ltd. 
A

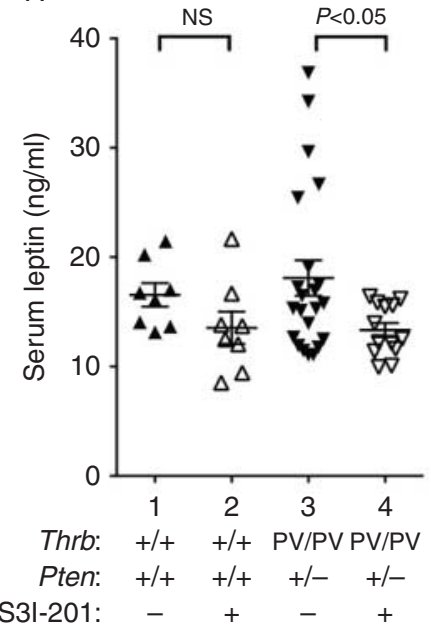

B-I

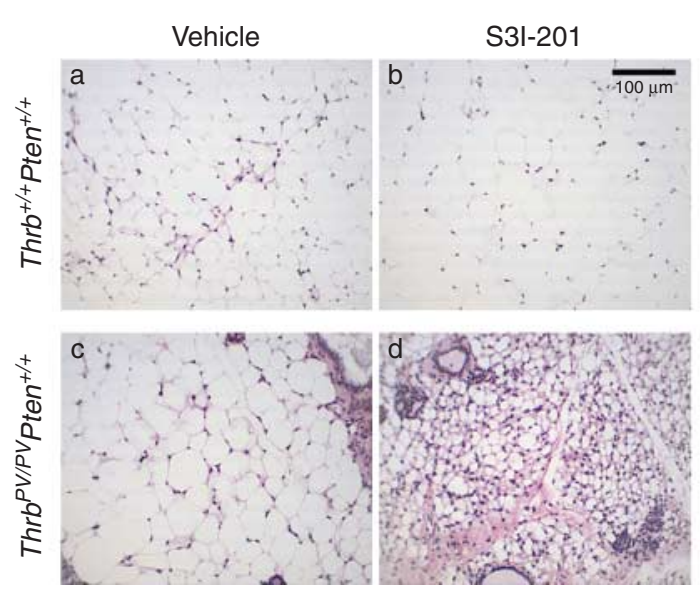

B-II

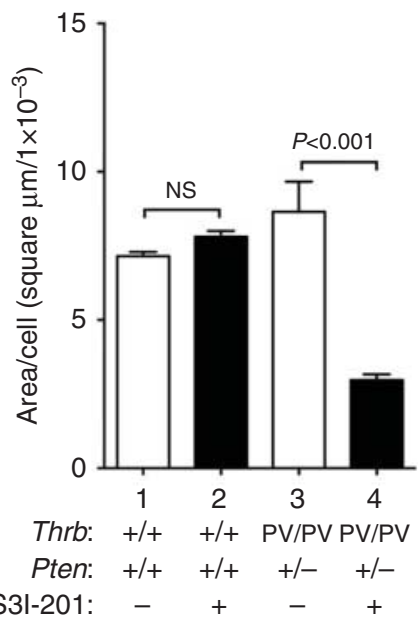

Figure 3

The effects of S3I-201 on serum leptin levels and size of adipocytes in the HFD-WT and HFD-Thrb ${ }^{P V I P V}$ Pten $^{+/-}$mice. (A) The serum leptin concentrations in WT or Thrb ${ }^{P V I P V}$ Pten $^{+/-}$mice were determined as described in Materials and methods. The serum leptin levels from WT mice treated with vehicle $(n=8)$ and S3I-201 $(n=8)$ were determined, as were the serum leptin levels from Thr $^{\text {PVIPV }}$ Pten $^{+/-}$mice treated with vehicle $(n=23)$ and S3I-201 ( $n=13)$. The $P$ values are indicated. NS, not significant.

in HFD-WT mice (lanes 1 and 2 vs lanes 3 and 4). In contrast, p-JAK2 protein levels were decreased by S3I-201 treatment in HFD-Thrb ${ }^{P V / P V}$ Pten $^{+/-}$mice (lanes 5 and 6 vs lanes 7 and 8, Fig. 4A-b), resulting from the decreased serum leptin levels. However, no effects of S3I-201 on the p-JAK2 was detected in HFD-WT mice (lanes 1 and 2 vs lanes 3 and 4) consistent with no changes in the serum leptin levels (Fig. 3A).
(B) Representative histological features of inguinal fat of HFD-WT and HFD$\mathrm{Thrb}^{\text {PVIPV }}$ Pten $^{+/-}$mice with or without S3I-201 (panel a, b, c, and d). (C) Fat cell size was measured for each genotype with vehicle or \$3I-201 shown in B. Data are expressed as mean \pm S.E.M. $(n=4)$. The $P$ values are shown. A full colour version of this figure is available at http://dx.doi.org/10.1530/ ERC-15-0417.

Consistently, the protein abundance of p-STAT3 was markedly decreased by inhibitor treatment (compare lanes 7 and 8 with lanes 5 and 6, Fig. 4A-d) without significant changes in the protein levels of total STAT3 (panel e, Fig. 4A). The reduction can be seen more clearly in the quantitative data shown in Fig. 4B-c in that the ratio of p-STAT3/total STAT3 was reduced by $90 \%$. These results indicate that S3I-201 effectively inhibited the activation of
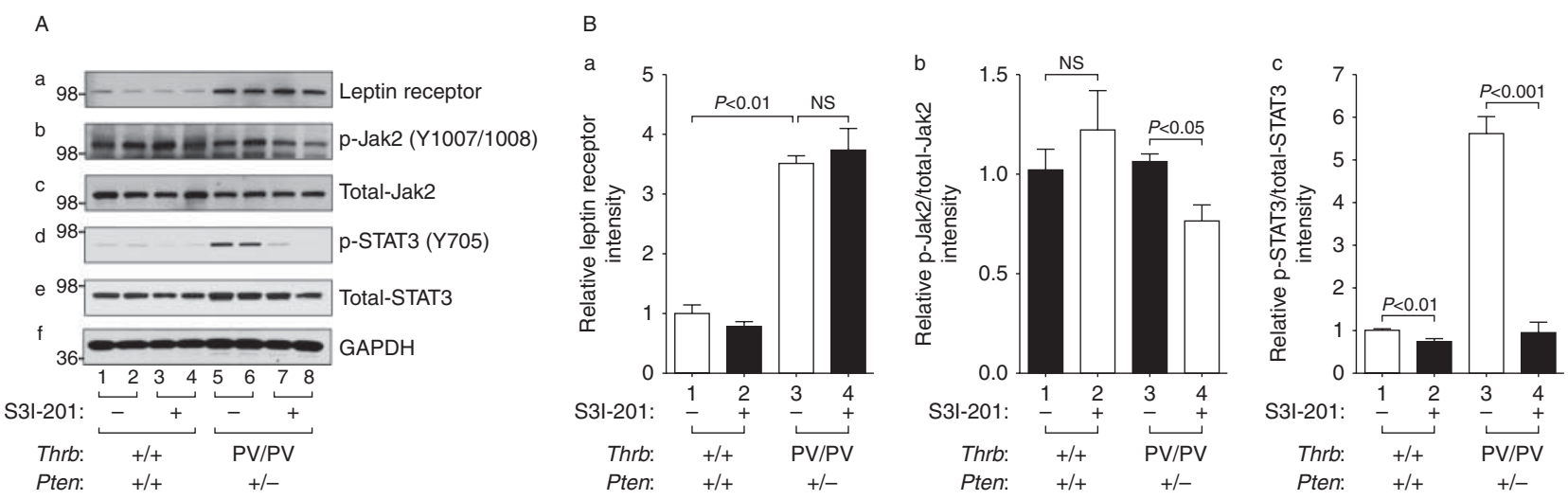

\section{Figure 4}

The effects of S3I-201 on protein levels of regulators in the STAT3 pathway in thyroids of HFD-WT and HFD-Thrb ${ }^{\text {PVIPV }}$ Pten $^{+/-}$mice. (A) Western blot analysis of protein abundance of leptin receptor, phosphorylated -JAK2 (Y1007/1008), total JAK-2, phosphorylated STAT3 (Y705), total-STAT3, and
GAPDH as a loading control after treatment with vehicle or S3I-201 in WT $(n=2)$ and Thrb ${ }^{\text {PVIPV }}$ Pten ${ }^{+1-}$ mice $(n=2)$. (B) The band intensities of the protein detected in (A) were quantified and compared. The data, shown as mean \pm S.E.M., were analyzed by Student's $t$-test. 
Jak2-STAT3 signaling in thyroid tumors of HFDThrb $^{\text {PV/PV }}$ Pten $^{+/-}$mice.

The decreased protein abundance of p-STAT3 detected by western blot was further confirmed by IHC analysis. Figure 5A-I shows the cells positively stained for the p-STAT3 in thyroid tumor cells HFD-Thrb ${ }^{P V / P V}$ Pten $^{+/-}$ mice or follicular cells of HFD-WT mice or with or without S3I-201 treatment. The reduction in the p-STAT3 positively stained cells was evident in mice treated with S3I-201 (Fig. 5A-I, panel h) as compared with mice treated with vehicle (Fig. 5A-I, panel f). The quantification shows $70 \%$ fewer cells were stained with p-STAT3 antibodies (Fig. 5A-II, bar 4 vs bar 3). A low signal of p-STAT3 was detected in the follicular cells of HFD-WT mice (Fig. 5A-I, panel b), which was reduced by S3I-201 (Fig. 5A-I, panel d; and A-II, bar 2 vs bar 1).

To delineate the functional consequences of inhibition of STAT3 activity by S3I-201 in HFD-Thrb ${ }^{P V / P V}$ Pten $^{+/-}$ mice, we used real-time qRT-PCR to compare the mRNA expression of STAT3 target genes in the thyroid tumors from S3I-201-treated and vehicle-treated groups. Five downstream target genes of STAT3 were repressed in thyroid tumors of HFD-Thrb ${ }^{P V / P V}$ Pten $^{+/-}$mice by S3I-201 (Fig. 5B). The reduced expression of $\mathrm{Ccnd1}, \mathrm{Myc}$, and $\mathrm{Bcl} 2$ genes both decreased proliferation and increased survival of tumor cells (Zhang et al. 2012). Socs3 (suppressor of cytokine signaling 3), induced by cytokines and leptin, and Mcl1, an STAT3 target gene that functions to mediate the actions of STAT3 in human cancer (Alvarez et al. 2005) were also repressed by S3I-201. Thus, inhibition of STAT3 activity led to the suppression of its downstream target genes to contribute to the delayed tumor progression in growth and survival.

We further investigated whether S3I-201 treatment could prevent aggressive cancer progression to distant metastasis in HFD-Thrb ${ }^{P V / P V}$ Pten $^{+/-}$mice. All HFD$\mathrm{Thrb}^{\text {PV/PV}}$ Pten $^{+/-}$mice treated with vehicle or S3I-201 developed the early-stage tumor phenotypes of hyperplasia and capsular invasion (Fig. 6A-I). However, the prevalences of vascular invasion and anaplasia in the S3I-201-treated group were less frequent than in vehicletreated HFD-Thrb ${ }^{P V / P V}$ Pten $^{+/-}$mice (Fig. 6A-I) at the same age. While $25 \%$ of vehicle-treated HFD-Thrb ${ }^{P V / P V}$ Pten $^{+/-}$ mice developed metastasis, no occurrence of metastasis was detected in inhibitor-treated HFD-Thrb ${ }^{P V / P V}$ Pten $^{+/-}$ mice at the same age. As shown by histopathological analysis (Fig. 6A-II, panel c), anaplasia was observed in the vehicle-treated HFD-Thrb ${ }^{P V / P V}$ Pten $^{+/-}$mice. In contrast, only earlier pathological changes of vascular invasion were detected in inhibitor-treated HFD-Thrb ${ }^{P V / P V}$ Pten $^{+/-}$ mice at the same age ( $\sim 5$ months of age). The development of lung metastasis was observed in the

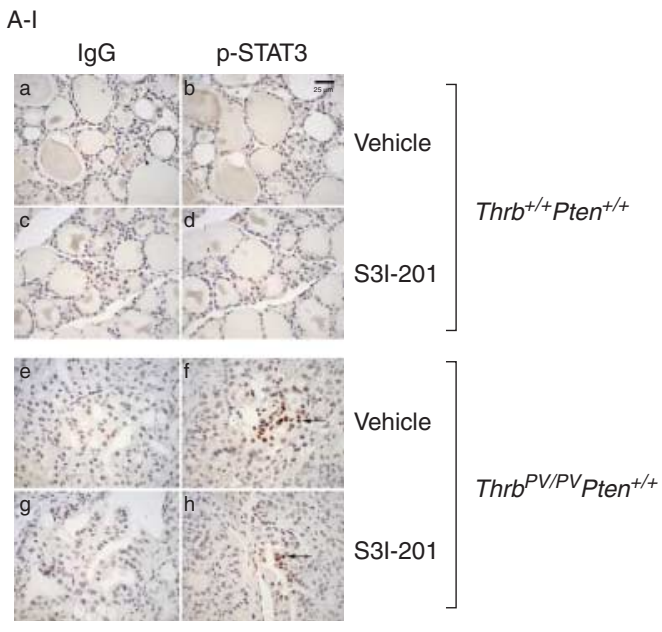

A-II

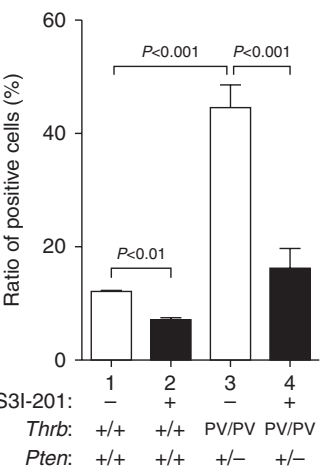

B

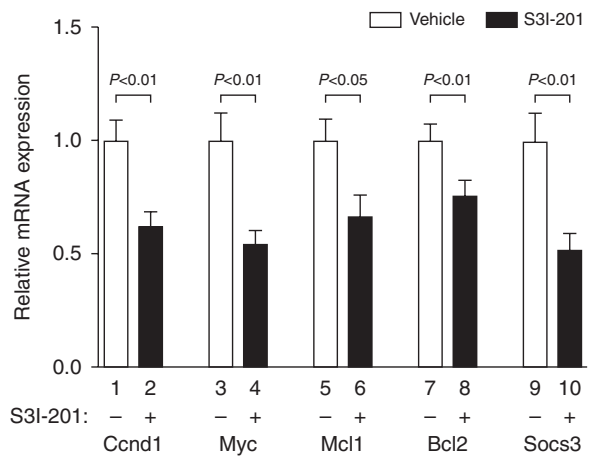

Figure 5

The effect of S3I-201 on STAT3 protein abundance in the thyroid of HFD-WT and HFD-Thrb ${ }^{\text {PVIPV }}$ Pten $^{+/-}$mice. (A-I) Comparison between the two groups of representative microphotographs of immunohistochemical analyses using anti-p-STAT3 antibody on thyroid sections from vehicle-treated or S3I-201-treated WT (panels $b$ and d) and Thrb ${ }^{\text {PVIPV }}$ Pten $^{+/-}$mice (panels $f$ and $h$ ). The negative controls from no primary antibodies are shown in the corresponding panels (WT; a and c, Thrb ${ }^{\text {PVIPV }}$ Pten $^{+\prime-}$ mice e and g). (A-II) The p-STAT3-positively stained cells were counted and the data are expressed as percentage of p-STAT3-positive cells vs total cells. The data are expressed as mean \pm s.E.M. ( $n=3$ slides). The $P$ values are shown. (B) The relative mRNA expression of $C c n d 1, M y c, M c 11, B c / 2$, and Socs3 in thyroid tumors from vehicle-treated $(n=4)$ and S3I-201-treated $(n=4)$ groups. The data, presented as mean \pm S.E.M., were analyzed by Student's $t$-test. A full colour version of this figure is available at http://dx.doi.org/10.1530/ ERC-15-0417. 
A-I

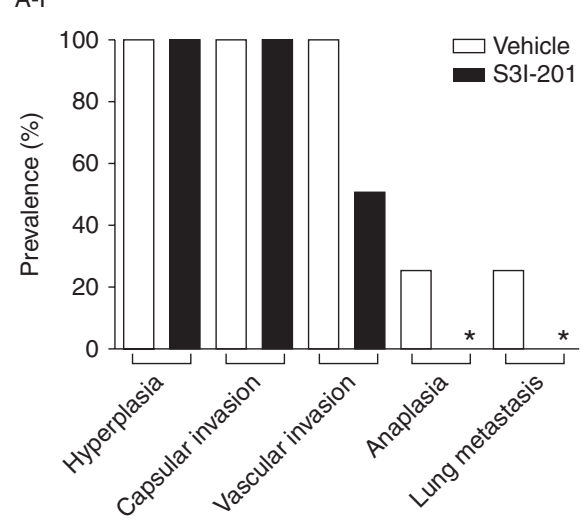

A-II

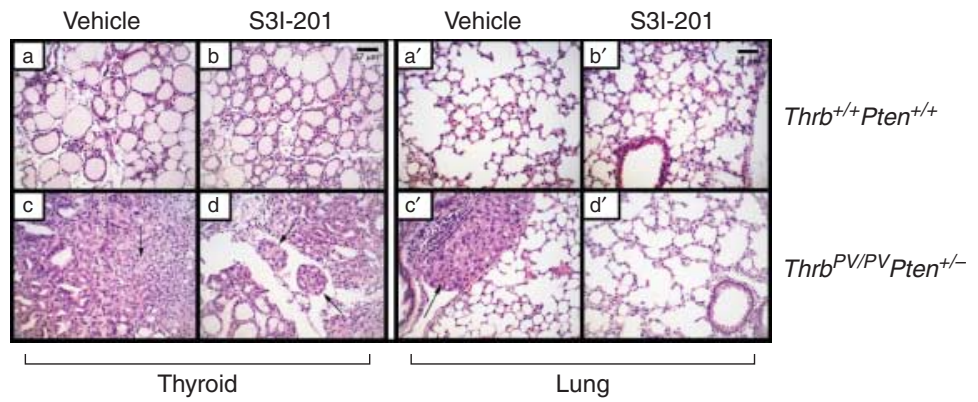

B-I

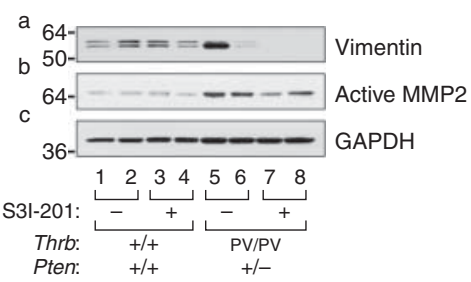

\section{Figure 6}

The effect of S3I-201 on pathological progression during thyroid carcinogenesis of HFD-Thrb ${ }^{\text {PVIPV }}$ Pten $^{+1-}$ mice. (A-I) Pathologic analysis in the vehicle-treated $(n=4)$ and S3I-201-treated $(n=4)$ groups of $\mathrm{Thrb}^{\text {PVIPV }}$ Pten $^{+/-}$mice. The prevalence of each pathological feature in thyroid carcinogenesis, according to the vehicle or \$3I-201 treatment, is shown a percentage. (A-II) Representative examples of hematoxylin and eosin (H\&E)-stained thyroid sections from WT mice treated with vehicle (panel a) and S3I-201 (panel b) and lung sections treated with vehicle (panel $a^{\prime}$ ) and S3I-201 (panel $b^{\prime}$ ) and thyroid tumor sections from

vehicle-treated group (Fig. 6A-II, panel $\mathrm{c}^{\prime}$ ) but not in the S3I-201-treated group in $\mathrm{Thrb}^{\mathrm{PV} / \mathrm{PV}} \mathrm{Pten}^{+/-}$mice at the same age (Fig. 6A-II, panel $\mathrm{d}^{\prime}$ ). In HFD-WT mice, no apparent effects of inhibitor on the histological features of the thyroid (panel a vs panel $b$ ) and the lung (panel $a^{\prime}$ vs panel $b^{\prime}$ ). These data indicate that S3I-201 could prevent metastasis in HFD-Thrb ${ }^{\text {PV/PV }}$ Pten $^{+/-}$mice.

To understand how S3I-201 delayed tumor invasion and prevented distal metastasis, we evaluated the protein abundance of key effectors of cancer metastatic signals downstream of STAT3 signaling. The migration of tumor cells through matrix barriers, tissue compartments, vessels, and organ boundaries is essential in the development of distant metastasis (Guarino 2010, Yadav et al. 2011, Lamouille et al. 2014, Tania et al. 2014). STAT3 is known to activate the expression of vimentin (Yadav et al. 2011, Xiong et al. 2012) and matrix-degrading proteases such as matrix metalloproteinase-2 (MMP-2), two critical effectors

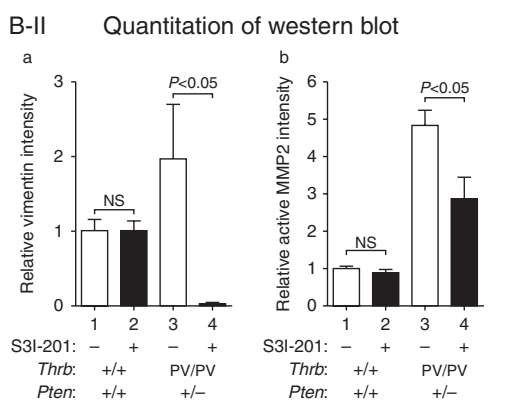

Thrb $^{\text {PVIPV }}$ Pten $^{+1-}$ mice treated with vehicle (panel c) and S3I-201 (panel d) and lung sections treated with vehicle (panel $c^{\prime}$ ) and S3I-201 (panel $d^{\prime}$ ). The arrows indicate vascular invasion (panel d) and anaplasia (panel c). (B-I) western blot analysis of vimentin, active MMP2 and GAPDH as a loading control after treatment with vehicle and S3I-201 in WT and Thrb $^{\text {PVIPV }}$ Pten $^{+1-}$ mice. (B-II) The band intensities of the protein detected in B-I were quantified and compared. The data, shown as mean \pm s.E.M., were analyzed by Student's $t$-test.

in the epithelial-mesenchymal transition (EMT) (Xie et al. 2004, Lamouille et al. 2014). We therefore evaluated the protein expression levels of vimentin and MMP-2 in thyroid tumors of HFD-Thrb ${ }^{P V / P V}{ }^{P_{t e n}{ }^{+/-}}$mice treated with vehicle or S3I-201. Figure 6B-I shows that the protein levels of vimentin were nearly totally inhibited by S3I-201 (Fig. 6B-I, panel a, lanes 7 and 8 vs lanes 5 and 6, bars 3 and 4, Fig. 6B-IIa) and that active MMP2 was markedly inhibited (40\%) (Fig. 6B-I, panel b, lanes 7 and 8 vs lanes 5 and 6; bars 3 and 4, Fig. 6B-IIb). Taken together, these data show that inhibition of STAT3 activity by S3I-201 was effective in delaying thyroid tumor progression and preventing distal metastasis in HFD-Thrb ${ }^{\text {PV/PV }}$ Pten $^{+/-}$mice.

\section{Discussion}

Recent epidemiological studies have shown that the incidence of thyroid cancer has rapidly increased in

Published by Bioscientifica Ltd. 
parallel with a dramatic rise in the prevalence of obesity (Simard et al. 2012). Moreover, extensive reports have provided compelling evidence to show a close association of obesity with increased thyroid cancer risk (Pappa \& Alevizaki 2014). Even so, the evidence to support a causal relationship of obesity with thyroid cancer is still lacking. Recently, we used a mouse model of thyroid cancer to show that HFD-induced obesity exacerbates thyroid cancer progression and distant metastasis (Kim et al. 2013b). Molecular analyses elucidate that the elevated serum leptin secreted by enlarged adipocytes in obese $\mathrm{Thrb}^{\mathrm{PV} / \mathrm{PV}} \mathrm{Pten}^{+/-}$mice leads to activation of leptin-JAK2STAT3 signaling to drive obesity-induced thyroid cancer progression (Kim et al. 2013b).

These findings provided the basis for us to seek further validation in a direct link of obesity with exacerbated thyroid cancer progression as indicated by increasing occurrence of vascular invasion and anaplasia, (i.e., dedifferentiation). Indeed, in the present studies, using an STAT3 specific inhibitor, S3I-201, we found that blocking the signaling initiated at the STAT3 node of the leptin-leptin receptor-JAK2-STAT3 pathway could delay cancer progression by inhibition of tumor cell proliferation and prevent metastasis by suppressing EMT. These results provided evidence for the first time to directly link obesity with advanced cancer progression. These results are consistent with the epidemiological findings in that aggressive thyroid cancer phenotypes of increased tumor size, extrathyroidal invasion, and advanced disease stage, are closely associated with increased BMI in patients (Kim et al. 2013a). Thus, the present molecular studies have further strengthened the causal relationship between obesity and thyroid cancer.

Because very little is known about whether the serum leptin could signal via the leptin receptor to activate STAT3 downstream signaling in the thyroid, we have carried out functional validation analysis by injecting recombinant leptin intraperitoneally to assess the activation of STAT3 by measuring p-STAT3 protein levels via immunohistochemical determination. Indeed, we detected increased p-STAT3 protein levels above the basal level in a time-dependent manner, indicating the leptin can exert its action in the thyroid (Kim et al. 2013b). In the present studies, we further detected higher leptin receptor protein levels in HFD-Thrb ${ }^{P / P V} \mathrm{Pten}^{+/-}$mice than in HFD-WT mice. Interestingly, the elevated leptin receptor was not affected by treatment of HFD$\mathrm{Thrb}^{\text {PV/PV }} \mathrm{Pten}^{+/-}$mice with the inhibitor (Fig. 4A), suggesting that the elevated serum leptin levels is most likely the initial driver in the activation of leptin-leptin
receptor-JAK2-STAT3 signaling, rather than also via the concurrent increases in the expression of the leptin receptor. Indeed, we found that S3I-201 significantly decreased the serum leptin levels of S3I-201 treated HFDThrb $^{\text {PV/PV }}$ Pten $^{+/-}$mice (Fig. 3A). STAT3 is known to express in human adipocytes (Harp et al. 2001), mouse adipocytes (Deng et al. 2006), and mouse 3T3-L1 cells (Stephens et al. 1996, Wang et al. 2010) to regulate adipogenesis. Activation of STAT3 activity leads to the induction of adipogenesis (Stephens et al. 1996, Harp et al. 2001, Wang et al. 2010) and inhibition of STAT3 activity by inhibitors or siRNA resulted in suppression of adipocyte differentiation (Deng et al. 2006, Wang et al. 2010). In line with these findings, we found that S3I-201 treated HFD$\mathrm{Thrb}^{P V / P V}$ Pten $^{+/-}$mice markedly decreased adipocyte cell size (Fig. 3B-I and -II), an indication of suppressed adipogenesis. As a result of decreased adipocytes, serum leptin levels were decreased (Fig. 3A). Thus, S3I-201 could act not only in the thyroid, but also in the adipocytes. S3I-201 acted directly on the adipocytes to attenuate the activated STAT3 activity in HFD-Thrb ${ }^{P V / P V}$ Pten $^{+/-}$mice to suppress adipogenesis, resulting in decreased leptin levels. The reduced leptin levels led to inhibit the activation of p-JAK2-STAT3 signaling in the thyroid to inhibit tumorgenesis.

Several recent reports have indicated a positive correlation of elevated serum leptin levels with differentiated thyroid carcinoma (Akinci et al. 2009, Rehem et al. 2014, Fan \& Li 2015). Moreover, elevated expression of leptin and/or leptin receptor in papillary thyroid cancer is associated with neoplasm aggressiveness, including tumor size and lymph node metastasis (Cheng et al. 2010). Recently, Lee et al showed the presence of an expanded intrathyroidal adipose depot and steatosis in thyroid epithelial cells in obese patients (Lee et al. 2015). While the intrathyroidal leptin levels from the expanded adipose depots are unknown, it is reasonable to assume that the intrathyroidal leptin levels would be elevated owing to the presence of an expanded intrathyroidal adipose depot in obese subjects. Thus, the findings from

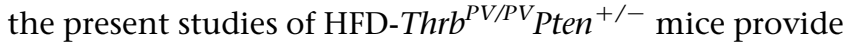
new mechanistic insights into the role of serum leptin in the carcinogenesis of the thyroid. Furthermore, together with the recent demonstration of intrathyroidal adipose depots in obese subjects (Lee et al. 2015), our studies raise the potential detrimental role of the intrathyroidal leptin in promoting thyroid cancer progression in obese patients.

STAT3 was originally identified as an oncogenic protein (Bromberg et al. 1999, Bowman et al. 2000) functioning to affect the expression of a wide array of

Published by Bioscientifica Ltd 
genes involved in apoptosis, cell migration, cell cycle regulation, angiogenesis, and modulation of immunosuppressive factors (Kim et al. 2014). Importantly, the upregulated STAT3 expression is associated with more malignant behavior of tumor cells and worse prognosis in a variety of human malignancies, such as cancer of lung, prostate, head and neck, ovarian, breast, gastric, pancreas, and thyroid (Wei et al. 2003, Masuda et al. 2007, Yakata et al. 2007, Sheen-Chen et al. 2008, Min \& Wei-hong 2009, Zhang et al. 2011, Ai et al. 2012, Liu et al. 2012, Dong et al. 2014). The increased expression and activation of STAT3 are detected in the tissue specimens of thyroid cancer, including lymphatic metastasis of papillary thyroid cancer in affected patients (Zhang et al. 2011, Dong et al. 2014). In view of the critical role of STAT3 in cancer progression, many STAT3 inhibitors are currently being tested in phase I and phase II clinical trials in patients with solid tumors such as brain tumors and melanomas (Dutzmann et al. 2015) and many types of leukemias and lymphomas (O'Shea et al. 2015). Currently, only limited clinical trials are assessing the effectiveness of STAT3 inhibitors in the treatment of thyroid cancer (Tsimberidou et al. 2009, Hong et al. 2012). Our preclinical studies suggest STAT3 inhibitors could be beneficial for obese thyroid cancer patients.

\section{Declaration of interest}

The authors declare that there is no conflict of interest that could be perceived as prejudicing the impartiality of the research reported.

\section{Funding}

This research was supported by the Intramural Research Program of the Center for Cancer Research, National Cancer Institute, National Institutes of Health.

\section{Author contribution statement}

J W Park and S Y Cheng developed the hypothesis, designed the experiments, wrote, and edited the manuscript. J W Park, C R Han, and $\mathrm{L}$ Zhao performed the experiments. M C Willingham contributed to the pathology review and interpretation.

\section{References}

Ai T, Wang Z, Zhang M, Zhang L, Wang N, Li W \& Song L 2012 Expression and prognostic relevance of STAT3 and cyclin D1 in non-small cell lung cancer. International Journal of Biological Markers 27 e132-e138. (doi:10. 5301/JBM.2012.9146)

Akinci M, Kosova F, Cetin B, Aslan S, Ari Z \& Cetin A 2009 Leptin levels in thyroid cancer. Asian Journal of Surgery/Asian Surgical Association 32 216-223. (doi:10.1016/S1015-9584(09)60397-3)
Alvarez JV, Febbo PG, Ramaswamy S, Loda M, Richardson A \& Frank DA 2005 Identification of a genetic signature of activated signal transducer and activator of transcription 3 in human tumors. Cancer Research $\mathbf{6 5}$ 5054-5062. (doi:10.1158/0008-5472.CAN-04-4281)

Aschebrook-Kilfoy B, Sabra MM, Brenner A, Moore SC, Ron E, Schatzkin A, Hollenbeck A \& Ward MH 2011 Diabetes and thyroid cancer risk in the National Institutes of Health-AARP Diet and Health Study. Thyroid 21 957-963. (doi:10.1089/thy.2010.0396)

Bowman T, Garcia R, Turkson J \& Jove R 2000 STATs in oncogenesis. Oncogene 19 2474-2488. (doi:10.1038/sj.onc.1203527)

Bromberg JF, Wrzeszczynska MH, Devgan G, Zhao Y, Pestell RG, Albanese C \& Darnell JE Jr 1999 Stat3 as an oncogene. Cell 98 295-303. (doi:10. 1016/S0092-8674(00)81959-5)

Cheng SP, Chi CW, Tzen CY, Yang TL, Lee JJ, Liu TP \& Liu CL 2010 Clinicopathologic significance of leptin and leptin receptor expressions in papillary thyroid carcinoma. Surgery 147 847-853. (doi:10.1016/ j.surg.2009.11.004)

Deng J, Hua K, Caveney EJ, Takahashi N \& Harp JB 2006 Protein inhibitor of activated STAT3 inhibits adipogenic gene expression. Biochemical and Biophysical Research Communications 339 923-931. (doi:10.1016/j.bbrc. 2005.10.217)

Dong W, Cui J, Tian X, He L, Wang Z, Zhang P \& Zhang H 2014 Aberrant sonic hedgehog signaling pathway and STAT3 activation in papillary thyroid cancer. International Journal of Clinical and Experimental Medicine 7 1786-1793.

Dutzmann J, Daniel JM, Bauersachs J, Hilfiker-Kleiner D \& Sedding DG 2015 Emerging translational approaches to target STAT3 signalling and its impact on vascular disease. Cardiovascular Research 106 365-374. (doi:10.1093/cvr/cvv103)

Fan YL \& Li XQ 2015 Expression of leptin and its receptor in thyroid carcinoma: distinctive prognostic significance in different subtypes. Clinical Endocrinology 83 261-267. (doi:10.1111/cen.12598)

Furumoto H, Ying H, Chandramouli GV, Zhao L, Walker RL, Meltzer PS, Willingham MC \& Cheng SY 2005 An unliganded thyroid hormone $\beta$ receptor activates the cyclin D1/cyclin-dependent kinase/retinoblastoma/E2F pathway and induces pituitary tumorigenesis. Molecular and Cellular Biology 25 124-135. (doi:10.1128/MCB.25.1.124-135.2005)

Guarino M 2010 Src signaling in cancer invasion. Journal of Cellular Physiology 223 14-26. (doi:10.1002/jcp.22011)

Guigon CJ, Zhao L, Willingham MC \& Cheng SY 2009 PTEN deficiency accelerates tumour progression in a mouse model of thyroid cancer. Oncogene 28 509-517. (doi:10.1038/onc.2008.407)

Harp JB, Franklin D, Vanderpuije AA \& Gimble JM 2001 Differential expression of signal transducers and activators of transcription during human adipogenesis. Biochemical and Biophysical Research Communications 281 907-912. (doi:10.1006/bbrc.2001.4460)

Hong DS, Kurzrock R, Supko JG, He X, Naing A, Wheler J, Lawrence D, Eder JP, Meyer CJ, Ferguson DA et al. 2012 A phase I first-in-human trial of bardoxolone methyl in patients with advanced solid tumors and lymphomas. Clinical Cancer Research 18 3396-3406. (doi:10.1158/10780432.CCR-11-2703)

Kim HJ, Kim NK, Choi JH, Sohn SY, Kim SW, Jin SM, Jang HW, Suh S, Min YK, Chung JH et al. 2013a Associations between body mass index and clinico-pathological characteristics of papillary thyroid cancer. Clinical Endocrinology 78 134-140. (doi:10.1111/j.1365-2265.2012. 04506.x)

Kim WG, Park JW, Willingham MC \& Cheng SY 2013 $b$ Diet-induced obesity increases tumor growth and promotes anaplastic change in thyroid cancer in a mouse model. Endocrinology 154 2936-2947. (doi:10.1210/en.2013-1128)

Kim JE, Patel M, Ruzevick J, Jackson CM \& Lim M 2014 STAT3 activation in glioblastoma: biochemical and therapeutic implications. Cancers 6 376-395. (doi:10.3390/cancers6010376)

Kitahara CM, Platz EA, Freeman LE, Hsing AW, Linet MS, Park Y, Schairer C, Schatzkin A, Shikany JM \& Berrington de Gonzalez A 2011 Obesity and thyroid cancer risk among US men and women: a pooled analysis of five 
prospective studies. Cancer Epidemiology, Biomarkers \& Prevention 20 464-472. (doi:10.1158/1055-9965.EPI-10-1220)

Lamouille S, Xu J \& Derynck R 2014 Molecular mechanisms of epithelialmesenchymal transition. Nature Reviews. Molecular Cell Biology 15 178-196. (doi:10.1038/nrm3758)

Lee MH, Lee JU, Joung KH, Kim YK, Ryu MJ, Lee SE, Kim SJ, Chung HK, Choi MJ, Chang JY et al. 2015 Thyroid dysfunction associated with follicular cell steatosis in obese male mice and humans. Endocrinology 156 1181-1193. (doi:10.1210/en.2014-1670)

Liu X, He Z, Li CH, Huang G, Ding C \& Liu H 2012 Correlation analysis of JAK-STAT pathway components on prognosis of patients with prostate cancer. Pathology Oncology Research 18 17-23. (doi:10.1007/s12253-0119410-y)

Masuda M, Ruan HY, Ito A, Nakashima T, Toh S, Wakasaki T, Yasumatsu R, Kutratomi Y, Komune S \& Weinstein IB 2007 Signal transducers and activators of transcription 3 up-regulates vascular endothelial growth factor production and tumor angiogenesis in head and neck squamous cell carcinoma. Oral Oncology 43 785-790. (doi:10.1016/j.oraloncology. 2006.10.007)

Meinhold CL, Ron E, Schonfeld SJ, Alexander BH, Freedman DM, Linet MS \& Berrington de Gonzalez A 2010 Nonradiation risk factors for thyroid cancer in the US Radiologic Technologists Study. American Journal of Epidemiology 171 242-252. (doi:10.1093/aje/kwp354)

Min H \& Wei-hong Z 2009 Constitutive activation of signal transducer and activator of transcription 3 in epithelial ovarian carcinoma. Journal of Obstetrics and Gynaecology Research 35 918-925. (doi:10.1111/j.14470756.2009.01045.x)

Nevins JR 1992 E2F: a link between the Rb tumor suppressor protein and viral oncoproteins. Science 258 424-429. (doi:10.1126/science. 1411535)

O'Shea JJ, Schwartz DM, Villarino AV, Gadina M, McInnes IB \& Laurence A 2015 The JAK-STAT pathway: impact on human disease and therapeutic intervention. Annual Review of Medicine 66 311-328. (doi:10.1146/annurev-med-051113-024537)

Pappa T \& Alevizaki M 2014 Obesity and thyroid cancer: a clinical update. Thyroid 24 190-199. (doi:10.1089/thy.2013.0232)

Rehem RA, Elwafa WA, Elwafa RA \& Abdel-Aziz TE 2014 Study of serum leptin in well-differentiated thyroid carcinoma: correlation with patient and tumor characteristics. World Journal of Surgery $\mathbf{3 8}$ 2621-2627. (doi:10.1007/s00268-014-2634-8)

Renehan AG, Tyson M, Egger M, Heller RF \& Zwahlen M 2008 Body-mass index and incidence of cancer: a systematic review and meta-analysis of prospective observational studies. Lancet 371 569-578. (doi:10.1016/ S0140-6736(08)60269-X)

Sen N, Che X, Rajamani J, Zerboni L, Sung P, Ptacek J \& Arvin AM 2012 Signal transducer and activator of transcription 3 (STAT3) and survivin induction by varicella-zoster virus promote replication and skin pathogenesis. PNAS 109 600-605. (doi:10.1073/pnas.1114232109)

Sheen-Chen SM, Huang CC, Tang RP, Chou FF \& Eng HL 2008 Prognostic value of signal transducers and activators of transcription 3 in breast cancer. Cancer Epidemiology, Biomarkers \& Prevention 17 2286-2290. (doi:10.1158/1055-9965.EPI-08-0089)

Siddiquee K, Zhang S, Guida WC, Blaskovich MA, Greedy B, Lawrence HR, Yip ML, Jove R, McLaughlin MM, Lawrence NJ et al. 2007 Selective chemical probe inhibitor of Stat3, identified through structure-based virtual screening, induces antitumor activity. PNAS 104 7391-7396. (doi:10.1073/pnas.0609757104)
Simard EP, Ward EM, Siegel R \& Jemal A 2012 Cancers with increasing incidence trends in the United States: 1999 through 2008. CA: A Cancer Journal for Clinicians 62 118-128. (doi:10.3322/caac.20141)

Stephens JM, Morrison RF \& Pilch PF 1996 The expression and regulation of STATs during 3T3-L1 adipocyte differentiation. Journal of Biological Chemistry 271 10441-10444. (doi:10.1074/jbc.271.18.10441)

Tania M, Khan MA \& Fu J 2014 Epithelial to mesenchymal transition inducing transcription factors and metastatic cancer. Tumour Biology $\mathbf{3 5}$ 7335-7342. (doi:10.1007/s13277-014-2163-y)

Tsimberidou AM, Vaklavas C, Wen S, Hong D, Wheler J, Ng C, Naing A, Tse S, Busaidy N, Markman M et al. 2009 Phase I clinical trials in 56 patients with thyroid cancer: the M D Anderson Cancer Center experience. Journal of Clinical Endocrinology and Metabolism 94 4423-4432. (doi:10.1210/jc.2009-0743)

Wang D, Zhou Y, Lei W, Zhang K, Shi J, Hu Y, Shu G \& Song J 2010 Signal transducer and activator of transcription 3 (STAT3) regulates adipocyte differentiation via peroxisome-proliferator-activated receptor gamma (PPAR $\gamma)$. Biology of the Cell/Under the Auspices of the European Cell Biology Organization 102 1-12. (doi:10.1042/BC20090070)

Wei D, Le X, Zheng L, Wang L, Frey JA, Gao AC, Peng Z, Huang S, Xiong HQ, Abbruzzese JL et al. 2003 Stat3 activation regulates the expression of vascular endothelial growth factor and human pancreatic cancer angiogenesis and metastasis. Oncogene 22 319-329. (doi:10.1038/ sj.onc.1206122)

Xie TX, Wei D, Liu M, Gao AC, Ali-Osman F, Sawaya R \& Huang S 2004 Stat 3 activation regulates the expression of matrix metalloproteinase-2 and tumor invasion and metastasis. Oncogene 23 3550-3560. (doi:10.1038/sj.onc.1207383)

Xiong H, Hong J, Du W, Lin YW, Ren LL, Wang YC, Su WY, Wang JL, Cui Y, Wang ZH et al. 2012 Roles of STAT3 and ZEB1 proteins in E-cadherin down-regulation and human colorectal cancer epithelial-mesenchymal transition. Journal of Biological Chemistry 287 5819-5832. (doi:10.1074/ jbc.M111.295964)

Yadav A, Kumar B, Datta J, Teknos TN \& Kumar P 2011 IL-6 promotes head and neck tumor metastasis by inducing epithelial-mesenchymal transition via the JAK-STAT3-SNAIL signaling pathway. Molecular Cancer Research 9 1658-1667. (doi:10.1158/1541-7786.MCR-11-0271)

Yakata Y, Nakayama T, Yoshizaki A, Kusaba T, Inoue K \& Sekine I 2007 Expression of p-STAT3 in human gastric carcinoma: significant correlation in tumour invasion and prognosis. International Journal of Oncology 30 437-442.

Zhang J, Gill A, Atmore B, Johns A, Delbridge L, Lai R \& McMullen T 2011 Upregulation of the signal transducers and activators of transcription 3 (STAT3) pathway in lymphatic metastases of papillary thyroid cancer. International Journal of Clinical and Experimental Pathology 4 356-362.

Zhang X, Yue P, Page BD, Li T, Zhao W, Namanja AT, Paladino D, Zhao J, Chen Y, Gunning PT et al. 2012 Orally bioavailable small-molecule inhibitor of transcription factor Stat 3 regresses human breast and lung cancer xenografts. PNAS 109 9623-9628. (doi:10.1073/pnas. 1121606109)

Zhao ZG, Guo XG, Ba CX, Wang W, Yang YY, Wang J \& Cao HY 2012 Overweight, obesity and thyroid cancer risk: a meta-analysis of cohort studies. Journal of International Medical Research 40 2041-2050. (doi:10.1177/030006051204000601)

Zhu X, Zhao L, Park JW, Willingham MC \& Cheng SY 2014 Synergistic signaling of KRAS and thyroid hormone receptor $\beta$ mutants promotes undifferentiated thyroid cancer through MYC up-regulation. Neoplasia 16 757-769. (doi:10.1016/j.neo.2014.08.003)

Received in final form 4 November 2015

Accepted 9 November 2015

Made available online as an Accepted Preprint

9 November 2015 http://erc.endocrinology-journals.org DOI: 10.1530/ERC-15-0417
(C) 2016 Society for Endocrinology Printed in Great Britain
Published by Bioscientifica Ltd 